\title{
A rare case of lens neovascularisation in retinoblastoma
}

\begin{abstract}
Retinoblastoma, the most common primary malignant intraocular tumor of childhood has been previously associated with neovascularisation of the iris and retina. New vessel formation in the crystalline lens is an extremely rare entity in association with retinoblastoma. In the present case, we describe such a case with new rosettes and histopathological high risk factors with detailed pathological and histological documentations.
\end{abstract}

Keywords: retinoblastoma, lens neovascularisation, new rosette
Volume 8 Issue 3 - 2018

Diva Kant Misra, Dipankar Das, Kasturi Bhattacharjee, Manabjyoti Barman, Panna Deka, Harsha Bhattacharjee, Sumega Singh Tomar,Apurba Kumar Deka Sri Sankaradeva Nethralaya Guwahati, India

Correspondence: Diva Kant Misra, Sri Sankaradeva Nethralaya, Beltola, Guwahati-781028, Assam, India, Tel + 9670964875, 036 I-2228879, 23055 I6, Fax \#036I2228878, Email divakant@gmail.com

Received: May 15, 2018 | Published:June 07, 2018

\section{Introduction}

Retinoblastoma $(\mathrm{Rb})$ is the most common primary intraocular tumor of the childhood occurring one in 18,000 live births. ${ }^{1}$ Group $\mathrm{E} \mathrm{Rb}$ comprises of extensive tumor occupying more than $50 \%$ of the globe or $\mathrm{Rb}$ associated with conditions like neovascular glaucoma, vitreous haemorrhage obscuring the tumor or significant hyphema and invasion of other ocular structures. ${ }^{2} \mathrm{Rb}$ can clinically present in varied ways and has been previously associated with neovascularisation of the iris and other ocular structures. ${ }^{3}$ But it is rarely reported in association with lens vascularisation. Here we describe a unique case of $\mathrm{Rb}$ associated with lens neovascularisation accompanied with pathological documentation.

\section{Case report}

A 4 month old female baby presented to the institute with leukocoria of right eye (OD) noticed by her parents. B-Scan ultrasound showed high reflective clumps of echoes in the vitreous cavity, suggestive of intra-tumoral calcifications. Magnetic resonance imaging of brain and orbit suggested $\mathrm{Rb}$ of $\mathrm{OD}$ with no obvious extraocular extension. Patient was diagnosed as group $\mathrm{E} \mathrm{Rb}$ of OD and enucleation and silicon ball implantation was done under general anaesthesia. The specimen was sent to the ocular pathology laboratory at the institute. After adequate fixation of the eyeball in $10 \%$ neutral buffered formalin, it was examined the next day. For fixation in $10 \%$ neutral buffered formalin, time period for total fixation is around 24 hours. $1 \mathrm{~mm}$ penetration of eyeball takes 1 hour, so $24 \mathrm{~mm}$ of eyeball will take roughly 24 hours. However, if extensive calcification is anticipated than it may require extended fixation time. The enucleated eyeball (Figure 1) measured anterio-posteriorly $19.07 \mathrm{~mm}$, horizontally $19.56 \mathrm{~mm}$ and vertically $18.15 \mathrm{~mm}$. Cornea measured horizontally $13.12 \mathrm{~mm}$ and vertically $11.23 \mathrm{~mm}$. The pupil was oval and measured $8.51 \mathrm{~mm}$. Optic nerve measured 9.11 in length and $4.06 \mathrm{~mm}$ in thickness. No transillumination defect was seen. No extra-scleral lesions were noted. Tumor measured $15.09 \mathrm{~mm}$ in height and $14.91 \mathrm{~mm}$ in basal diameter. The globe was sectioned vertically. A whitish endophytic tumor occupying most of the vitreous cavity with specks of calcification was seen. Lateral calottes were cut separately in broad loaf technique and were submitted for histopathology. Separate transverse section of the distal end of the optic nerve was cut and submitted for evaluation. Gross documentation was done.

Transparent crystalline lens was separately examined and showed neovascularisation (Figure 2a-d). Enucleated eye ball showed tumour occupying almost entire vitreous cavity. Crystalline lens was seen separately which looked slight yellow and transparent. Three blood vessels were seen in the lens in the peripheral edge and were documented under background illumination. Portion of the lens was again dissected and haematoxyline \& eosin (H\&E) stain was carried out and it revealed regressed blood vessels in the cross section. Haematoxylin was put on the slide for 10-15 minutes followed by a wash in the running water, then one dip is made in $1 \%$ acid alcohol, slide was washed followed by addition of $0.4 \%$ of ammonia for 1 minute. Third wash was made. Eosin was put on the slide for 1 minute and further washing is carried out. Xylene was added. Slide was dried and mounted for microscopic viewing. Study of the specimen in H\&E stain showed normal cornea with focal iris neovascularisation. An endophytic tumor was seen in the vitreous cavity with numerous Flexner Wintersteiner (FW), Homer Wright (HW) and few new true rosettes ${ }^{4}$ (Figure 3 ). Zones of necrosis seen without any calcifications. Few vesicular cells along with basophilic undifferentiated cells and occasional apoptotic bodies were noted. ${ }^{5}$ Neovascularization of lens was also documented in H\&E stained eyeball (Figure 4). Few pigment migrations were also noted. Exudations were seen in the section. Lateral calottes showed superficial involvement of the retinal pigment epithelium (RPE). The sclera was normal. Cut end of the optic nerve did not show tumor involvement. Immuno-Histochemisty (IHC) of the tumor was done for nueron specific enolase (NSE), glial fibrillary acidic protein (GFAP), p53, BCL2, transforming growth factor $\beta$ (TGF- $\beta$ ). NSE and p53 were positive in the tumor sample and others were negative. GFAP, BCL2, TGF beta were negative in the lenticular portion of the eyeball as well as the adjoining basophilic tumour zone. Patient was reviewed by an oncologist at the regional cancer centre (Figure 5). 


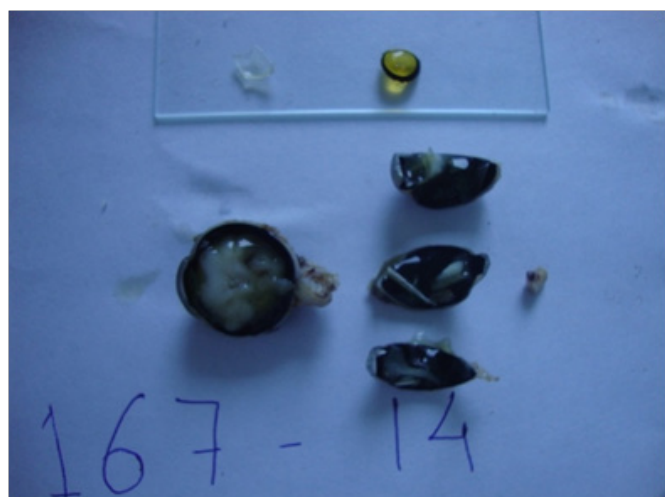

Figure I Gross documentation of the retinoblastoma with lateral calottes in broad loaf technique. The crystalline lens seen separately in the figure.

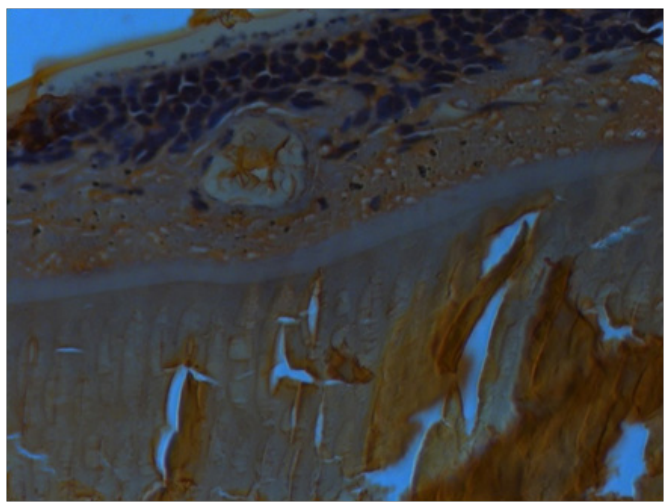

Figure 3 H\&E stain showing a vessel on the posterior surface of the lens (X200)

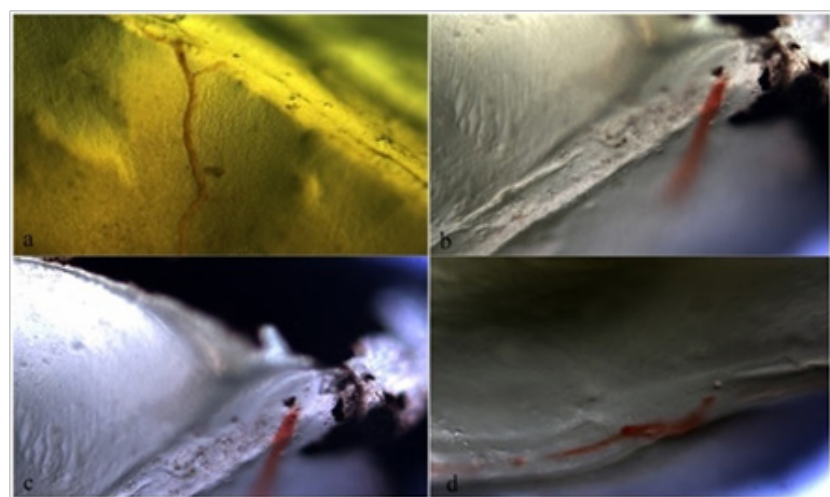

Figure 2 Gross examination of the crystalline lens showed blood vessels on the posterior capsule and stretched to the posterior surface of the lens

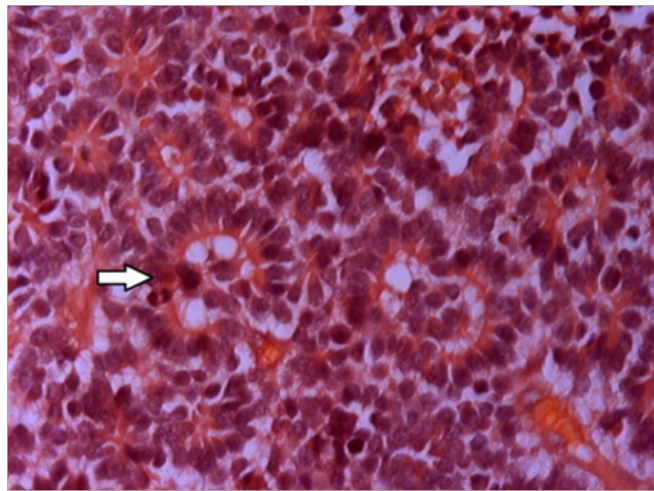

Figure 4 New rosettes (white arrow) along with FW \& HW rosettes were seen in the section in H\&E stain (X200).

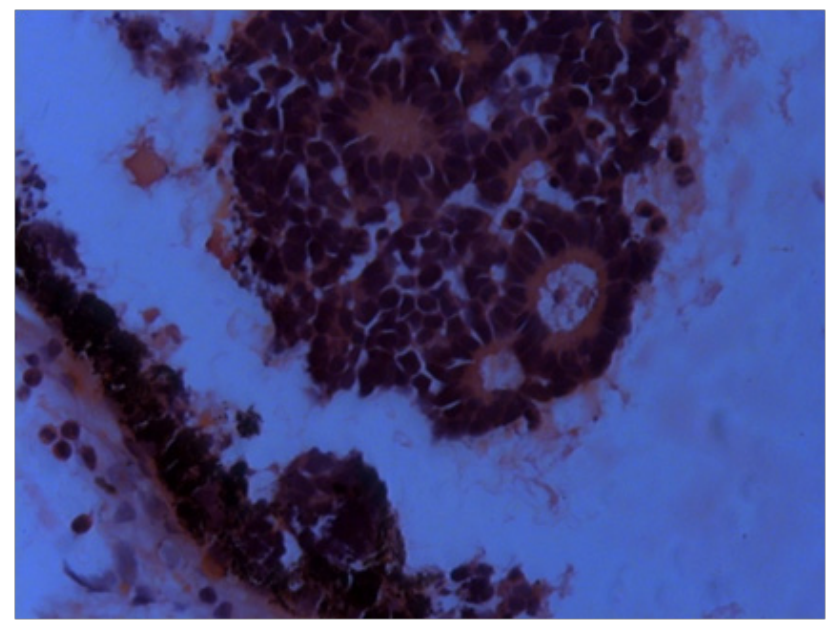

Figure $5 \mathrm{~A}$ cluster of tumor cells encroaching the RPE in H\&E stain (X200).

\section{Discussion}

$\mathrm{Rb}$ is the most common primary intraocular tumor of the childhood and has diverse presentations. Neovascularisation of the lens is a rare entity and has been previously described in association with proliferative diabetic retinopathy and retinal detachment. ${ }^{6,7} \mathrm{We}$ documented focal neovascularisation of lens in association with retinoblastoma. Subsequent thorough search of literature revealed that no case of neovascularisation of lens has been ever reported with $\mathrm{Rb}$. A natural balance of angiogenic and anti-angiogenic factor is crucial for the eye to prevent the formation of any form of neovascularisation.
Intraocular tumours like $\mathrm{Rb}$ have the potential to upset this natural balance resulting in angiogenesis. Hypoxic conditions within the $\mathrm{Rb}$ may lead to neovascularisation of the lens. The lens capsule secretes anti-endothelial cell inhibitory factors inhibiting neovascularisation of the lens even in the presence of concurrent iris neovascularisation. ${ }^{8}$ Any micro breach in the lens capsule allows it to respond to lens specific growth factors to permit proliferation of new vessels. Additionally the RPE is known to secrete inhibitors of vascular endothelial cell growth. ${ }^{9}$ RPE involvement in this particular case may be a contributing factor. 
Neovascularisation of lens has to be differentiated from remnant of tunica vasculosa lentis where the hyaloid artery enters the eye at the optic disc and then branches to form a network of capillaries on the posterior surface of the lens capsule. At approximately 9 weeks gestation, the capillary network surrounding the lens is fully developed and it disappears by an orderly process of programmed cell death shortly before birth. In our case, there was no pathological evidence of Mittendorf dot or pupillary membrane or strands. Here, we had seen differentiated RB with FW, HW and the new rosettes described by us. ${ }^{4}$ The newer rosettes were evolving one and they were associated with histopathological high risk factors such as focal rubeosis and superficial RPE involvement. ${ }^{10}$ These findings stressed that newer rosettes may be strong indication to mark the bad prognosis in RB. ${ }^{4}$ Positive IHC for TGF- $\beta$ is an important indicator of a hypoxic condition. We wanted to co-relate for the hypoxic condition of the lens and its surrounding. However we could not find co-relation with it. Our findings suggest that it is important to consider the possibility of neovascularisation of other ocular structures apart from the iris and retina. Tumor biology for $\mathrm{Rb}$ with neovascularisation may be an important issue that researchers should undertake for further validation of hypoxic condition induced by the tumor and its changes.

\section{Acknowledgements}

Sri Kanchi Sankara Health and Educational Foundation.

\section{Conflict of interest}

The author declares that there is no conflict of interest.

\section{References}

1. Bishop JO, Madson EC. Retinoblastoma. Review of the current status.
Surv Oophthalmol. 1975;19(6):342-366.

2. Shields CL, Mashayekhi A, Au AK, et al. The international classification of retinoblastoma predicts chemoreduction success. Ophthalmology. 2006;113(12):2276-2280.

3. Shields JA, Shields CL. Retinoblastoma: clinical and pathologic features. In: Shields JA, Shields CL, editors. Intraocular tumors. An atlas and textbook, $1^{\text {st }}$ ed. Philadelphia: Saunders, 1992. p. 305-332.

4. Das D, Bhattacharjee K, Barthakur SS, et al. A new rosette in retinoblastoma. Indian J Ophthalmol. 2014;62(5):638-641.

5. Das D, Bhattacharjee H, Bhattacharjee K. Efferocytosis in Retinoblastoma. Journal of cancer therapy. 2013;4:1443-1447.

6. Comarrata MR, Chang S, Sparrow J. Iris neovascularization in proliferative vitreoretinopathy. Ophthalmology. 1992;99(6):898-905.

7. Barile GR, Chang S, Horowitz JD, et al. Neovascular complications associated with rubeosis iridis and peripheral retinal detachment after retinal detachment surgery. Am J Ophthalmol. 1998;126(3):379-389.

8. Williams GA, Eisenstein R, Schumacher B, et al. Inhibitor of vascular endothelial cell growth in the lens. Am J Ophthalmol. 1984;97(3):366371.

9. Glaser BM, Campochiaro PA, Davis JL. Retinal pigment epithelial cells release inhibitors of neovascularisation. In. Benezra D, Ryan N, Glaser $\mathrm{BM}$, et al. editors. Ocular circulation and neovascularisation, $1^{\text {st }}$ ed. Netherlands. Springer; 1987: 535-543.

10. Peer J, Neufeld M, Baras M, et al. Rubeosis iridis in retinoblastoma. Histologic findings and the possible role of vascular endothelial growth factor in its induction. Ophthalmology. 1997;104(4):1251-1258. 